\title{
\#MeTooOrthopaedics: A protocol to determine the prevalence of gender-based and sexual harassment in the field of orthopaedic surgery
}

\author{
Zoe Bond ${ }^{1}$, Patricia Schneider ${ }^{1}$, Kim Madden ${ }^{2}$, Sheila Sprague ${ }^{1.2}$, Paula McKay $^{2}$, Anthony \\ Bozzo $^{1}$, Mohit Bhandari ${ }^{1,2}$, Michelle Ghert ${ }^{1,3}$ \\ ${ }^{1}$ Department of Surgery, McMaster University, Hamilton, Ontario, Canada \\ ${ }^{2}$ Department of Health Research Methods, Evidence, and Impact, McMaster University, \\ Hamilton, Ontario, Canada \\ ${ }^{3}$ Juravinski Cancer Centre, Hamilton Health Sciences, Hamilton, Ontario, Canada
}

\author{
Corresponding Author \\ Michelle Ghert, MD, FRCSC \\ Department of Surgery | McMaster University \\ 711 Concession Street, B3 Surgical Offices 169A \\ Hamilton, ON L8V 1C3 \\ ghertm@mcmaster.ca \\ T: 905-387-9495 ext. 64089
}

Subject Areas: Orthopaedics, Science and Medical Education, Surgery and Surgical Specialties, Women's Health

Keywords: Orthopaedic surgery, gender-based harassment, sexual harassment, prevalence, survey, research methods 
2 Background. Research shows that gender-based and sexual harassment are prevalent within 3 training and practicing medical establishments, with differences across specific specialties. 4 Orthopaedic surgery remains a male-dominated field, with recent statistics reporting women 5 representing only 5\% and $12 \%$ of orthopaedic surgeons in the United States and Canada, 6 respectively. This represents a significant gender discrepancy and, although there may be many reasons why this discrepancy exists, it is justified to explore whether gender-based and sexual harassment may contribute to this disparity.

Objectives. This study aims to determine the prevalence of gender-based and sexual harassment in orthopaedic surgery, and to determine the impact of these experiences on female orthopaedic surgeons. It will also explore the association of such gender-based and sexual harassment experiences with certain demographic factors.

Methods. We will conduct a cross-sectional survey of female orthopaedic surgeons, fellows and residents that are current members of a participating orthopaedic society. We have developed a unique questionnaire to gather information concerning gender-based and sexual harassment experiences of female orthopaedic surgeons, fellows and residents throughout their education and current practices. The questionnaire will be administered online using SurveyMonkey®, the online survey tool, to ensure anonymity. Reminder emails will be distributed up to two times after the survey is initially distributed to maximize the number of responses and, thus, validity and generalizability. Descriptive analyses and multivariable logistic regression analyses will be conducted to analyze the collected data.

Conclusions. The results of this study are likely to bring to light a critical issue in orthopaedic surgery and will hopefully provide the impetus for orthopaedic departments and societies to develop and enforce policies that limit these destructive behaviors in the workplace. We hope the results will provide sufficient information to determine if these experiences are one of the factors leading to the pronounced gender disparity within this field.

Ethics and Dissemination. An ethics application is currently under review with the Hamilton Integrated Research Ethics Board (HiREB) in Hamilton, ON, Canada. The results of this initiative will be disseminated through peer-reviewed publications and conference presentations. 


\section{Study Summary}

- The primary objectives of this study will be to determine the prevalence of gender-based and sexual harassment among female orthopaedic surgeons, fellows and residents over the course of their training and professional careers.

- Although this topic has been explored in different medical fields, this protocol represents the first investigation in the field of orthopaedic surgery.

- We plan to collect data anonymously to minimize apprehension to participate and to produce accurate and representative data.

- We expect that including participants across all career stages and broad geographic regions will produce more generalizable results.

- The experiences of participants may differ from those who decline participation. Individuals may be less likely to participate if they are a victim of gender-based or sexual harassment, thus resulting in a lower prevalence rate. Conversely, some individuals may opt not to participate because they are not victims of gender-based or sexual harassment and, as such, feel that the issue is not relevant to them, thereby overstating the prevalence. 


\section{Introduction}

There have been several recent high-profile movements led by women who have publicly reported their experiences with gender-based and sexual harassment. Due to such movements, many individuals have found the courage to come forward with their personal, similar experiences.

These movements, however, have focused mainly on high-profile workplaces, such as politics, television and movies, and have yet to extend to the "average" everyday workplaces. This study will focus on gender-based and sexual harassment in the field of orthopaedic surgery, which remains one of the highest male-dominated specialties in the medical field (Canadian Medical Association, 2016).

\section{What are Gender-Based and Sexual Harassment?}

The Ontario Human Rights Commission (OHRC) defines gender-based harassment as "any behavior that polices and reinforces traditional heterosexual gender norms", such as, for example, stereotypes of the dominant male and subservient female (OHRC, 2013). Division XV.1 of Part III of the Canada Labour Code defines sexual harassment as "any conduct, comment, gesture, or contact of a sexual nature that is likely to cause offence or humiliation to any employee; or that might, on reasonable grounds, be perceived by that employee as placing a condition of a sexual nature on employment or on any opportunity for training or promotion." (Government of Canada, 2016)

\section{The Burden of Gender-Based and Sexual Harassment on the Workplace}

Gender-based and sexual harassment that occurs in the workplace is not only detrimental to the person who has experienced the harassment, but also to the workplace as a whole. The World Health Organization (WHO) recently outlined their global strategy for health workers, which aims to eliminate gender-based violence, discrimination and harassment from health-related workforces by enforcing the personal and professional rights of all health workers (including freedom from all kinds of discrimination, coercion and violence) (WHO, 2016). If employers do not take the necessary actions to deter these behaviors within the workplace, many consequences may materialize. The obvious consequences fall upon those who experience harassment, as they may be subjected to embarrassment or even assault, which may lead to the inability to effectively meet the expectations of the job and, therefore, fail to reach their full potential or result in a reduction in earnings. (OHRC, n.d.) Gender-based and sexual harassment may also result in barriers to entry or integration into the desired position or field, or the need to alter aspirations in order to feel safe and comfortable within the workplace. Unimpeded gender-based and sexual harassment within the workplace may also result in incidentals for the employer; such as health or legal expenses, absence or loss of good workers, decreased productivity, and an overall low morale (OHRC, n.d.).

\section{Trends in Medicine and Surgery}

Research shows that gender-based and sexual harassment are prevalent within training and practicing medical establishments, with variance based on specific specialties. In 2016, Jagsi et al. reported that $30 \%$ of women disclosed experiencing sexual harassment in the academic 
medical setting, as did $4 \%$ of men. Stratton et al. (2005) reported that exposure to gender discrimination and sexual harassment influenced the choices of almost 50\% of women, and $16.4 \%$ of men, in their pursuit of a medical specialty. Specifically, Stratton et al. (2005) reported that $56 \%$ of men who chose obstetrics and gynecology in their residency selection experienced gender discrimination and / or sexual harassment, as did 38\% of women. This data suggests that everyone can be affected by gender and sexual harassment, and that even though obstetrics and gynecology is a female-dominated field, over one-third of women still experienced harassment. It, therefore, follows that male-dominated fields are likely to present even greater challenges to women in the workplace with respect to gender-based and sexual harassment.

\section{Gender-Based and Sexual Harassment in Orthopaedic Surgery}

Orthopaedic surgery is a significantly male-dominated profession (Canadian Medical Association, 2017; Association of American Medical Colleges, 2016). While there are other surgical specialties with a large gender disparity, orthopaedics has remained exceptionally sparse in female representation (Baumhauer \& Pinzur, 2014). Recent North American statistics indicate that only $12 \%$ and $5 \%$ of orthopaedic surgeons in Canada and the United States, respectively, are women (Canadian Medical Association, 2017; Association of American Medical Colleges, 2016). This represents a significant gender discrepancy and, although there may be many reasons why this discrepancy exists, it is justified to explore whether gender-based and / or sexual harassment play a role in this disparity as this may warrant additional resources to manage victims or changes in policies to prevent such destructive behavior in the orthopaedic workplace.

\section{Rationale for the Present Study}

Previous research has shown that gender-based and sexual harassment are prevalent in medical professions, including female-dominated specialties, which provides rationale for the investigation of strongly male-dominated medical specialties. Although the proportion of total female physicians has greatly increased since the 1950s (AMN Healthcare, 2015), proportionate growth within orthopaedic surgery has not occurred and there is no conclusive data in the literature to justify this enduring gender gap. We aim to quantify the prevalence of gender-based and sexual harassment within orthopaedic surgery, and to determine whether these behaviors have contributed to the stagnation of female representation within the field. Our rationale for surveying surgeons at different stages of their careers is to capture any differences that may exist between different generations and time points in careers. There is a lack of evidence on this topic in general, but especially within orthopaedic surgery. We aim to address this lack of knowledge and increase awareness, which may direct future policies to deter further gender-based and sexual harassment in the orthopaedic surgery workplace.

\section{Primary Research Question}

What is the prevalence of both gender-based and sexual harassment over course of the training and professional careers of female orthopaedic surgeons, fellows and residents? 


\section{Research Objectives \& Hypotheses}

The primary objectives of this survey will be to: A) determine the prevalence of gender-based harassment among female orthopaedic surgeons, fellows, and residents over the course of their training and professional careers; and B) determine the prevalence of sexual harassment among female orthopaedic surgeons, fellows, and residents over the course of their training and professional careers. For the purposes of our study, we have defined gender-based and sexual harassment as follows:

- Gender-based harassment occurs when a person experiences discrimination or unequal treatment based on his / her gender or gender identity. The harassment does not need to be based on anything of a sexual nature. Instead, gender-based harassment usually involves stereotypes based on the traditional roles and functions associated with a gender.

- Sexual harassment is bullying or coercion of a sexual nature, or the unwelcome or inappropriate promise of rewards in exchange for sexual favors. Such harassment can include unwelcome sexual advances, requests for sexual favors, and other verbal or physical harassment of a sexual nature.

The secondary objectives of this survey will be to: A) identify demographic factors, if any, that are associated with experiencing gender-based harassment; and B) identify demographic factors, if any, that are associated with experiencing sexual harassment.

We hypothesize that the prevalence of gender-based and sexual harassment experienced by female orthopaedic surgeons, fellows, and residents throughout their education and professional careers is sufficiently high enough to warrant the development of policies (or the enforcement of existing ones) that address this issue and aim to minimize gender-based and sexual harassment behaviors.

\section{Research Methods \& Materials}

We will conduct an international cross-sectional survey of female orthopaedic surgeons, fellows and residents, wherein 300 women will complete a self-reported web-based questionnaire across orthopaedic societies in North America, with the intention of expanding to other international societies. The reporting of our final results will adhere to the guidelines ascribed by the Checklist for Reporting Results of Internet E-Surveys (CHERRIES) reporting checklist.

\section{Eligibility Criteria}

We will consider individuals that meet all the following criteria eligible for the study: A) the individual identifies as female; B) the individual is either an orthopaedic resident, fellow or staff surgeon; C) the individual is a current member of a participating orthopaedic society; and D) the individual is able to read and write in English. 


\section{Intervention}

\section{Survey Development}

We have developed a unique questionnaire to assess the prevalence of gender-based and sexual harassment within orthopaedic surgery, specifically targeting female orthopaedic surgeons, fellows and residents. Our questionnaire is an adaptation of the pre-existing Sexual Experiences Questionnaire (SEQ), Sexual Experiences Questionnaire-Department of Defense (SEQ-DoD), and the Survey on Sexual Misconduct in the Canadian Armed Forces so that we can further explore gender-based and sexual harassment specific to our study population (Fitzgerald et al., 1995; Fitzgerald et al., 1999; Statistics Canada, 2016). The development of our questionnaire was also informed by a review of the current literature and other published gender-based and sexual harassment surveys, and in consultation with intimate partner violence researchers who have previously surveyed female patients on a similarly sensitive topic.

The draft questionnaire was reviewed by three additional health research methodology experts to ensure that: A) nothing vital was missing; B) the wording of the questions is clear, precise and addresses the objectives of the study (content validity); and $\mathrm{C}$ ) the overall perception is that the questionnaire has face validity, addressing the issues in question. The questionnaire was then piloted with select female surgeons from other specialties to confirm face and content validity. Finally, the questionnaire was revised based upon the recommendations of this group of experts and re-tested until no additional issues or concerns were identified.

\section{Survey Design}

The questionnaire is comprised of 90 items, and consists of Likert-scale questions, checkboxes, as well as two brief open-ended questions. The questionnaire is sub-divided into four sections described as follows: A) Demographics and Current Education / Work Details; B) Harassment in the Workplace (separated into two parts [Part I: Gender-Based Harassment; and Part II: Sexual Harassment]); C) Behavior Details; and D) Final Thoughts and Opinions. All questions are straightforward and utilize clear and widely recognized terminology to enhance the validity of results. The original survey length has been minimized to capture only the necessary information to fulfill the study objectives, aiming to increase the overall response rate and limit barriers that would affect its proper completion.

\section{Primary Outcome Measures}

To measure the prevalence of both gender-based and sexual harassment, our questionnaire will ask participants whether they have experienced a variety of behaviors widely considered to be either gender-based or sexual harassment over the course of their training and professional careers (see Table One). To estimate the overall prevalence of gender-based harassment, we will combine the positive answers to all questions on gender-based harassment behaviors. We believe that various forms of sexual behaviors - verbal, emotional and physical - are equally destructive and often concomitant. Therefore, to estimate the overall prevalence of sexual harassment, we will combine the positive answers to all questions pertaining to sexual harassment behaviors, irrespective of whether they are of a verbal, emotional or physical nature. 


\section{Survey Administration}

The questionnaire will be administered to all female members of the Canadian Orthopaedic Organization (COA) and the Canadian Orthopaedic Residents' Association (CORA), with the intention of expanding to other international orthopaedic societies. We will compile an email distribution list of those who meet the inclusion criteria and distribute the questionnaire according to standard methodology practice for a cross-sectional survey study. Reminder emails will be distributed up to two times after the survey is initially distributed to maximize the number of responses and, thus, validity and generalizability. These reminders will be sent in three-week intervals, meaning the survey will close to further responses nine weeks after the initial distribution. Financial compensation will not be provided for completing the questionnaire.

Completion of the survey will be entirely voluntary; no written informed consent will be pursued, as consent is implied upon submission of the questionnaire. Participants will be informed that the survey is completely anonymous and that their personal answers will not be shared. Participants will also be advised to find a private location where they can complete the questionnaire to ensure privacy and anonymity, thereby maximizing the quality of their responses.

\section{Rationale for the Study Design}

A self-administered online questionnaire will be our primary data collection tool. The selfadministered survey method has been shown in multiple studies to be generally favoured over other survey methods due to various forms of selection and information bias (Bowling, 2005). While the use of in-person interviews has been thought to achieve substantially higher response rates than self-completed questionnaires, participants often overstate their responses to please the interviewer (Goyder, 1985). Thus, this self-administered online questionnaire aims to eliminate response bias as there is no interviewer for the respondent to "impress". Furthermore, in-person interviews are not well suited for this study due to the sensitive nature of the topic and the desire for anonymity. Sampling from several national orthopaedic societies that include orthopaedic surgeons in both academic and non-academic settings should account for sampling bias, thereby ensuring that the results of this study are representative of female orthopaedic surgeons from diverse cultures. The online nature of this questionnaire aims to eliminate procedural bias, as the participants can choose a convenient time to complete the questionnaire either in part, or in its entirety, thereby eliminating any pressure or time restraints allowing them to satisfactorily reflect on their experiences and provide as much detail as they feel necessary.

\section{Data Collection}

We will use the SurveyMonkey® online survey tool to develop and distribute the online questionnaire. This platform has several useful features, including the ability to track survey completion by participant name while maintaining anonymity of questionnaire responses, thereby allowing us to determine who has completed the survey with no method of linking a participant's name to her specific responses. This feature is important to decrease the nuisance of reminder emails to participants that have already completed the questionnaire, and to ensure each participant only completes the questionnaire once. Furthermore, considering the demands on the time of orthopaedic surgeons, we will ensure the online survey allows progress to be saved 
before final submission. This will ensure that participants do not have to complete the entire questionnaire in one sitting and can return to finish it at a later time (if they are using the same device to complete the questionnaire). Finally, due to the sensitive nature of this study, the online survey will have no requirements pertaining to the number of questions participants need to answer, thereby providing participants the opportunity to leave a question blank if they do not feel comfortable enough to answer.

\section{Strategies to Maximize Participation}

Due to the sensitive nature of the topic in question, some potential participants may be hesitant to participate. In addition, those who have not had any relevant experiences may not participate as they may feel that they do not have anything valuable to contribute. However, maximizing the response rate is important to meet the required sample size and to increase the generalizability of the results. In an effort to maximize participation, a detailed but concise Study Information Sheet will be distributed alongside the questionnaire. This document will provide sufficient information to all potential participants explaining the rationale for the study, the importance of participation (the ultimate goals of the project), the anonymity process, and the potential benefits and (unlikely) costs as a result from participation. Furthermore, the questionnaire will be distributed with several reminders at pre-determined intervals to encourage participation. Finally, the questionnaire has been revised and refined several times to ensure it is as short and concise as possible to minimize participant burden.

\section{Statistical Analysis Plan}

\section{Sample Size}

To determine the number of respondents needed to sufficiently power our analysis, we estimate that approximately $75 \%$ of female orthopaedic surgeons have, at one-point, experienced genderbased in their education or careers. McNamara et al. (1995) reported that approximately $70 \%$ of female emergency medical residents experienced gender-based harassment over the course of their education. Given the larger gender disparity, we expect that gender-based harassment is more pervasive in orthopaedic surgery than in other medical and surgical fields. Therefore, the calculation for an appropriate sample size was performed using the following formula, and assuming a $95 \%$ confidence interval:

where:

$$
N=\left(z^{2} \times(p[1-p])\right) / w^{2}
$$

$\mathrm{N}=$ required sample size

$\mathrm{z}=\mathrm{z}$ value ( 1.96 for $95 \%$ confidence interval)

$\mathrm{w}=$ half the width confidence interval, expressed as a decimal $(0.05= \pm 5)$

$\mathrm{p}=$ hypothesized proportion of female orthopaedic surgeons that experience / have experienced gender-based harassment in the workplace, expressed as a decimal $(75 \%=$ $0.75)$

$$
N=288
$$

McNamara et al. (1995) also reported that approximately 65\% of female emergency medical residents experienced sexual harassment over the course of their education. Again, given the larger gender disparity, we expect that sexual harassment is more pervasive in orthopaedic 
surgery and, therefore, estimate that $70 \%$ of female orthopaedic surgeons have, at one-point, experienced sexual harassment in their education and careers. Thus, an appropriate sample size using the above formula, and assuming a 95\% confidence interval:

where:

$\mathrm{N}=$ required sample size

$\mathrm{z}=\mathrm{z}$ value ( 1.96 for $95 \%$ confidence interval)

$\mathrm{w}=$ half the width confidence interval, expressed as a decimal $(0.05= \pm 5)$

$\mathrm{p}=$ hypothesized proportion of female orthopaedic surgeons that experience / have experienced sexual harassment in the workplace, expressed as a decimal $(70 \%=0.70)$

$$
N=323
$$

Accordingly, 323 completed questionnaires will be required to facilitate a meaningful analysis of both primary outcomes. We have decided to increase the computed required sample size by $10 \%$ to account for missing data, bringing the total to $\underline{\mathbf{3 5 5}}$ completed questionnaires. There are approximately 1,000 female orthopaedic surgeons in North America, thus providing sufficient participation potential to recruit the proposed sample size within the study timeline (Canadian Medical Association, 2017; Association of American Medical Colleges, 2016).

\section{Statistical Methods}

Data will be analyzed using SPSS Version 25.0 [SPSS, Inc. | Chicago, IL, USA]. Demographic characteristics and participants' responses will be summarized descriptively as frequencies and percentages (for categorical variables) or mean/median with standard deviations depending on the distribution (for continuous variables). The primary outcomes (prevalence of gender-based and sexual harassment) will be reported as a numerator, denominator, and percentage with associated 95\% confidence interval (see Table Two). For the secondary analysis, we will conduct a multivariable logistic regression analysis on the following demographic characteristics to determine if any of these variables are independently associated with experiencing either gender-based and sexual harassment over the course of a participant's education and career: age (continuous), ethnicity, marital status, geographic region, career stage, and orthopaedic subspecialty (see Table Three). No statistical imputation process will be conducted to estimate missing data. A p value less than 0.05 will be considered statistically significant for all analyses.

\section{Ethics}

An ethics application is currently under review with the Hamilton Integrated Research Ethics Board (HiREB) in Hamilton, ON, Canada. All eligible individuals for this study will be provided sufficient information to make an informed decision about their participation in this study. Written informed consent will not be pursued for this study, as passive consent will be implied by completion and submission of the questionnaire and, thus, having their anonymized responses included in future iterations and analyses.

Information regarding study participants will be managed according to the requirements of the HiREB. Specifically, all personal data will be available to only the study's Project Manager and will be kept strictly confidential as it will only be used only to maintain a record of completion. Anonymized data will be accessible only to the study team, and will be destroyed after ten years, 
as per local guidelines. All data will be maintained on a password-protected computer on a secure network and in a locked office.

\section{Discussion}

The necessity for such a study is twofold. First, while previous studies have documented the gender imbalance in orthopaedic surgery, few have explored any real explanation with specific, measurable outcomes, and none, to our knowledge, have explicitly addressed the possibility of gender-based and sexual harassment contributing to the disparity. Second, gender-based and sexual harassment have the astronomical potential to negatively impact individuals and their careers, as well as the reputations of institutions and training programs. Although progress has been made, it is important to understand whether gender-based and sexual harassment exist in orthopaedic surgery in order to implement the appropriate policies so that the field can capitalize on the increased female population and growing diversity in medical school.

Our proposed study has several strengths. The study design is structured and transparent. Our unique questionnaire is clear, thorough and well-informed. To maximize the feasibility of the study and the generalizability of the results, we plan to survey female orthopaedic surgeons, fellows and residents from a broad range of geographic regions, education systems and career stages. It is hoped that the anonymity process we plan to employ will also maximize participation.

However, some limitations must also be recognized. A bias towards the under-reporting of gender-based and sexual harassment is our primary concern as participants may answer the questionnaire differently if they know that they could be identified as victims of gender-based or sexual harassment. The anonymity process during the data collection and analysis phases, as previously described, has been implemented to reduce this bias. Nevertheless, despite this consideration, it is still likely that under-reporting bias will exist - therefore, if our study finds an appreciable prevalence of gender-based and sexual harassment among female orthopaedic surgeons, fellows and residents, it is likely that the "true" prevalence is even greater.

In contrast, the opposite is also possible. Some individuals may opt not to participate because they are not victims of gender-based or sexual harassment and, as such, feel that the issue is not relevant to them, thereby overstating the prevalence of gender-based and sexual harassment in orthopaedic surgery. Because of this possibility, individuals will be instructed upon invitation (via the Study Information Sheet) that participation is important, even if the questions have no bearing on their lives.

Another potential source of bias is the self-administered and open nature of the questionnaire, which may result in some missing or inconsistent data. Due to the extremely sensitive and private information that we are asking participants to disclose, we believe that other survey techniques (such as interviewer-administered) or requiring participants to answer all questions is inappropriate. We have, however, a priori marginally increased our required sample size from what is necessary to still adequately power our primary analysis. 
An inherent limitation of this study design that could result in bias is the possibility of nonrandom differences between respondents and non-respondents for which we cannot account. Nonetheless, demographic data will be collected and analyzed so that we can discern whether the results are widely generalizable to the field of orthopaedic surgery.

Finally, while unlikely, the choice to participate in this study could incite anger in male peers. We will minimize any potential for harm by respecting the anonymity of each participant and affirming to her that her choice to participate or not participate in the study will never be disclosed, even to members of the study team other than the Project Manager. Furthermore, Dearwater et al. (1998) noted in their survey to determine the prevalence of intimate partner abuse in an emergency room setting that, as a safety precaution, women were only administered a questionnaire while in the absence of family, friends and other medical staff. We plan to adopt this practice for our survey by providing instructions for participants at the beginning of the questionnaire that the survey should be completed only when alone and in a private location.

\section{Conclusion}

Instances of gender-based and sexual harassment in high-profile workplaces are coming to light at a rapid pace. Evidence for such harassment in the medical field exists but has not been extensively studied. Although previous studies have postulated reasons for gender disparity in orthopaedic surgery (i.e., lifestyle choices), none have addressed the issue of gender-based and sexual harassment. The results of this study are likely to bring to light a critical issue in orthopaedic surgery and are likely to be of interest to orthopaedic departments and professional societies that are interested in promoting female representation in the field. We hope that the results will provide the impetus for these orthopaedic departments and societies to develop action plans, enforce policies and explore changes to existing policies to limit these destructive behaviors in the workplace.

\section{Dissemination Plan}

We expect that the results of this study will be published in a peer-reviewed journal. Furthermore, the findings of this study will also be disseminated to regional, national and international audiences through presentations at relevant research conferences.

\section{Competing Interests}

We have no competing interests to disclose.

\section{Author Contributions}

MG is the Principal Investigator. ZB, PS, AB and MG are responsible for the conception and design of the study, and the development of the study protocol. KM, SS, PM, AB and MB have contributed to the design of the study and the content of the study protocol with critical intellectual revisions. ZB and PS are responsible for drafting the protocol manuscript. All authors have read and approved the final manuscript. 


\section{$382 \quad$ Funding Statement}

383 We have received no funding for this study or the preparation of this manuscript. We disclose

384 receipt of the following research support: Dr. Mohit Bhandari is funded, in part, by a Canada

385 Research Chair (McMaster University). Dr. Michelle Ghert is funded, in part, by the Canadian

386 Institutes of Health Research and the Canadian Cancer Society Research Institute. These funding

387 sources had no role in the design of this study or the development of the study protocol. 


\section{References}

AMN Healthcare. 2015. Women in Medicine: A Review of Changing Physician Demographics, Female Physicians by Specialty, State and Related Data. Retrieved from https://www.amnhealthcare.com/uploadedFiles/MainSite/Content/Staffing_Recruitment/Staffcare-WPWomen\%20in\%20Med.pdf

Association of American Medical Colleges. 2016. Active Physicians by Sex and Specialty, 2015. Retrieved from https://www.aamc.org/data/workforce/reports/458712/1-3-chart.html

Baumhauer, J. F., \& Pinzur, M. S. 2014. Women in Orthopaedic Surgery. Foot \& Ankle International; 35(9): 949 950. doi:10.1177/1071100714534183

Bowling A. Mode of questionnaire administration can have serious effects on data quality. J Public Health 2005; 27(3): 281-91.

Canadian Medical Association. 2016. Canadian Specialty Profiles. Retrieved from https://www.cma.ca/En/Pages/specialty-profiles.aspx

Canadian Medical Association. 2017. Number and percent distribution of physicians by specialty and sex, Canada 2017. Retrieved from https://www.cma.ca/Assets/assets-library/document/en/advocacy/06-spec-sex.pdf

Dearwater SR, Coben JH, Campbell JC, Nah G, Glass N, McLoughlin E, Berkemeir B. 1998. Prevalence of intimate partner abuse in women treated at community hospital emergency departments. JAMA; 280(5): 433-8.

Fitzgerald, L. F., Gelfand, M. J., \& Drasgow, F. 1995. Measuring Sexual Harassment: Theoretical and Psychometric Advances. Basic and Applied Social Psychology; 17(4): 425-445. Retrieved from https://pdfs.semanticscholar.org/46a7/39a2bebc254a5c3583ffd16907a3a1f4feaf.pdf

Fitzgerald, L. F., Magley, V. J., Drasgow, F., \& Waldo, C. R. 1999. Measuring Sexual Harassment in the Military: The Sexual Experiences Questionnaire (SEQ-DoD). Military Psychology; 11(3): 243-263.

Government of Canada. 2016. Sexual Harassment. Retrieved from https://www.canada.ca/en/employment-socialdevelopment/services/labour-standards/reports/sexual-harassment.html

Goyder J. 1995. Face-to-face interviews and mailed questionnaires: the net difference in response rate. Public Opinion Quarterly; 49(2): 234-52.

Jagsi R, Griffith KA, Jones R, Perumalswami CR, Ubel P, Stewart A. 2016. Sexual Harassment and Discrimination Experiences of Academic Medical Faculty. JAMA; 315(19):2120-2121. doi:10.1001/jama.2016.2188

McNamara, R. M., Whitley, T. W., Sanders, A. B., \& Andrew, L. B. 1995. The extent and effects of abuse and harassment of emergency medicine residents. The SAEM In-service Survey Task Force. Academic Emergency Medicine; 2(4): 293-301.

Ontario Human Rights Commission. 2013. Policy on preventing sexual and gender-based harassment. Retrieved from http://www.ohrc.on.ca/en/policy-preventing-sexual-and-gender-based-harassment-0

Ontario Human Rights Commission. n.d. Sexual harassment in employment (fact sheet). Retrieved from http://www.ohrc.on.ca/en/sexual-harassment-employment-fact-sheet

Statistics Canada. 2016. Survey on Sexual Misconduct in the Canadian Armed Forces. Retrieved from http://www23.statcan.gc.ca/imdb/p3Instr.pl?Function=assembleInstr\&a=1\&\&lang=en\&Item_Id=285179

Stratton, T. D., McLaughlin, M. A., Witte, F. M., Fosson, S. E., \& Nora, L. M. 2005. Does Students' Exposure to Gender Discrimination and Sexual Harassment in Medical School Affect Specialty Choice and Residency Program Selection? Academic Medicine; 80(4): 400-408.

World Health Organization. 2016. Global strategy on human resources for health: Workforce 2030. Retrieved from http://apps.who.int/iris/bitstream/handle/10665/250368/9789241511131-

eng.pdf;jsessionid=02329D433381CA36C083D3300050053F? sequence $=1$ 
Table One: Gender-Based and Sexual Harassment Behavior Matrix

\begin{tabular}{|c|c|c|}
\hline Harassment Type & Behaviors / Related Questions & $\begin{array}{c}\text { Branching Questions? } \\
\text { (YES / NO) }\end{array}$ \\
\hline \multicolumn{3}{|c|}{ Gender-Based Harassment } \\
\hline Verbal / Psychological & $\begin{array}{l}\text { Q50. Have you ever been in a situation where a supervisor, colleague or patient treated you 'differently' because of } \\
\text { your gender (i.e., mistreated, slighted or ignored you)? } \\
\text { Q51. Have you ever been in a situation where a supervisor, colleague or patient told offensive jokes or remarks? } \\
\text { Q52. Have you ever been in a situation where a supervisor, colleague or patient made sexist comments or displayed } \\
\text { sexist materials (such as suggesting that people of your sex are not suited for the kind of work you do)? } \\
\text { Q53. Have you ever been in a situation where a supervisor, colleague or patient put you down or was condescending } \\
\text { to you because of your sex? } \\
\text { Q54. Have you ever been in a situation where a supervisor, colleague or patient suggested that you don't act like a } \\
\text { woman is supposed to act? } \\
\text { Q55. Have you ever been in a situation where you felt you received an inferior learning / work experience due to } \\
\text { your sex (such as a decreased number of opportunities, being held to a higher standard than male peer, etc.)? }\end{array}$ & $\begin{array}{l}\text { Q50. YES } \\
\text { Q51. YES } \\
\text { Q52. YES } \\
\text { Q53. YES } \\
\text { Q54. YES } \\
\text { Q55. YES }\end{array}$ \\
\hline Physical & N/A & N/A \\
\hline \multicolumn{3}{|c|}{ Sexual Harassment } \\
\hline Verbal / Psychological & $\begin{array}{l}\text { Q60. Have you ever been in a situation where a supervisor, colleague or patient made crude sexual remarks? } \\
\text { Q61. Have you ever been in a situation where a supervisor, colleague or patient attempted to establish a romantic } \\
\text { sexual relationship with you despite your attempts to discourage him/her (such as continuing to ask you for dates, } \\
\text { drinks, dinner, etc., even though you said 'No')? } \\
\text { Q62. Have you ever been in a situation where a supervisor, colleague or patient gave you unwanted attention (such } \\
\text { as whistles, calls, suggestive looks, gestures or body language)? } \\
\text { Q63. Have you ever been in a situation where a supervisor, colleague or patient told suggestive stories or displayed } \\
\text { suggestive materials? } \\
\text { Q64. Have you ever been in a situation where a supervisor, colleague or patient made inappropriate sexual } \\
\text { comments about your appearance or body? } \\
\text { Q69. Have you ever been in a situation where you felt you were being subtly bribed with some sort of reward (e.g., } \\
\text { preferential treatment) to engage in sexual behavior with a supervisor, colleague or patient? } \\
\text { Q70. Have you ever been in a situation where a supervisor, colleague or patient made you feel threatened with some } \\
\text { sort of retaliation for not being sexually co-operative (such as by mentioning an upcoming review)? } \\
\text { Q71. Have you ever been in a situation where you actually experienced negative consequences for refusing to } \\
\text { engage in sexul activity with a supervisor, colleague or patient? } \\
\text { Q72. Have you ever been in a situation where a supervisor or colleague made you feel afraid you would be treated } \\
\text { poorly in your learning / work environment if you didn't sexually co-operate? } \\
\text { Q73. Have you ever been in a situation where a supervisor or colleague implied faster promotions or better } \\
\text { treatment in your learning / work environment if you were sexually co-operative? }\end{array}$ & $\begin{array}{l}\text { Q60. YES } \\
\text { Q61. YES } \\
\text { Q62. YES } \\
\text { Q63. YES } \\
\text { Q64. YES } \\
\text { Q69. YES } \\
\text { Q70. YES } \\
\text { Q71. YES } \\
\text { Q72. YES } \\
\text { Q73. YES }\end{array}$ \\
\hline Physical & $\begin{array}{l}\text { Q65. Have you ever been in a situation where a supervisor, colleague or patient made physical contact that made } \\
\text { you feel uncomfortable (such as hugs or shoulder rubs or getting too close)? } \\
\text { Q66. Have you ever been in a situation where a supervisor, colleague or patient made unwanted attempts to stroke, } \\
\text { fondle or kiss you? } \\
\text { Q67. Have you ever been in a situation where a supervisor, colleague or patient unsuccessfully attempted to have } \\
\text { sex with you without your consent or against your will? } \\
\text { Q68. Have you ever been in a situation where a supervisor, colleague or patient had sex with you without your } \\
\text { consent or against your will? }\end{array}$ & $\begin{array}{l}\text { Q65. YES } \\
\text { Q66. YES } \\
\text { Q67. YES } \\
\text { Q68. YES }\end{array}$ \\
\hline
\end{tabular}


Table Two: Summary of Primary Outcome Analysis Plan

\begin{tabular}{|c|c|c|c|}
\hline Objective & Type of Variable & Source of Data & Method of Analysis \\
\hline $\begin{array}{l}\text { To determine the prevalence of } \\
\text { gender-based harassment }\end{array}$ & Binary & $\begin{array}{l}\text { Section B: Part One } \\
\text { (Questions } 50-55 \text { ) }\end{array}$ & $\begin{array}{c}\text { Descriptive } \\
\text { Numerator and denominator, } \\
\text { and percentage, with } 95 \% \\
\text { confidence interval }\end{array}$ \\
\hline $\begin{array}{l}\text { To determine the prevalence of total } \\
\text { sexual harassment }\end{array}$ & Binary & $\begin{array}{l}\text { Section B: Part Two } \\
\text { (Questions 60-73) }\end{array}$ & $\begin{array}{c}\text { Descriptive } \\
\text { Numerator and denominator, } \\
\text { and percentage with } 95 \% \\
\text { confidence interval }\end{array}$ \\
\hline $\begin{array}{l}\text { To determine the prevalence of each } \\
\text { gender-based harassment behavior } \\
\text { (see Table One) }\end{array}$ & Binary & $\begin{array}{l}\text { Section B: Part One } \\
\text { (Questions } 50-55 \text { ) }\end{array}$ & $\begin{array}{c}\text { Descriptive } \\
\text { Numerator and denominator, } \\
\text { and percentage with } 95 \% \\
\text { confidence interval }\end{array}$ \\
\hline $\begin{array}{l}\text { To determine prevalence of each } \\
\text { sexual harassment behavior (see Table } \\
\text { One) }\end{array}$ & Binary & $\begin{array}{l}\text { Section B: Part Two } \\
\text { (Questions } 60-73 \text { ) }\end{array}$ & $\begin{array}{c}\text { Descriptive } \\
\text { Numerator and denominator, } \\
\text { and percentage with } 95 \% \\
\text { confidence interval }\end{array}$ \\
\hline
\end{tabular}

Table Three: Summary of Secondary Analysis Plan

\begin{tabular}{|c|c|c|c|}
\hline Objective & Type of Variable & Source of Data & Method of Analysis \\
\hline $\begin{array}{l}\text { To determine if any of the following } \\
\text { variables are independently associated } \\
\text { with experiencing gender-based } \\
\text { harassment: age, ethnicity, marital } \\
\text { status, geographic region, career stage } \\
\text { and orthopaedic sub-specialty. }\end{array}$ & $\begin{array}{c}\text { Age: Continuous } \\
\text { Ethnicity: Categorical } \\
\text { Marital Status: Categorical } \\
\text { Geographic Region: } \\
\text { Categorical } \\
\text { Career Stage: Categorical } \\
\text { Sub-specialty: Categorical }\end{array}$ & $\begin{array}{c}\text { Age: Section A } \\
\text { (Question 1) } \\
\text { Ethnicity: Section A (Question } \\
\text { 2) } \\
\text { Marital status: Section A } \\
\text { (Question 3) } \\
\text { Geographic Region: Section A } \\
\text { (Questions 7, 15, 16, 28, 37, } \\
\text { 44) } \\
\text { Career Stage: Section A } \\
\text { (Questions 6, 29) } \\
\text { Sub-specialty: Section A } \\
\text { (Questions 14, 43) }\end{array}$ & $\begin{array}{l}\text { Multivariable logistic } \\
\text { regression analysis }\end{array}$ \\
\hline $\begin{array}{l}\text { To determine if any of the following } \\
\text { variables are independently associated } \\
\text { with experiencing sexual harassment: } \\
\text { age, ethnicity, marital status, } \\
\text { geographic region, career stage and } \\
\text { orthopaedic sub-specialty. }\end{array}$ & $\begin{array}{c}\text { Age: Continuous } \\
\text { Ethnicity: Categorical } \\
\text { Marital Status: Categorical } \\
\text { Geographic Region: } \\
\text { Categorical } \\
\text { Career Stage: Categorical } \\
\text { Sub-specialty: Categorical }\end{array}$ & $\begin{array}{c}\text { Age: Section A } \\
\text { (Question 1) } \\
\text { 2) } \\
\text { Ethnicity: Section A (Question } \\
\text { Marital status: Section A } \\
\text { (Question 3) } \\
\text { Geographic Region: Section A } \\
\text { (Questions 7, 15, 16, 28, 37, } \\
\text { 44) } \\
\text { Career Stage: Section A } \\
\text { (Questions 6, 29) } \\
\text { Sub-specialty: Section A } \\
\text { (Questions 14, 43) }\end{array}$ & $\begin{array}{l}\text { Multivariable logistic } \\
\text { regression analysis }\end{array}$ \\
\hline
\end{tabular}

